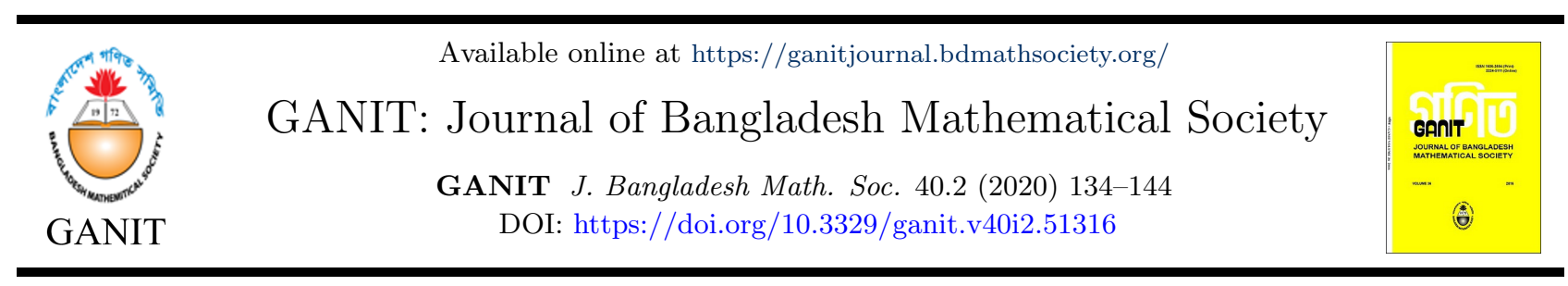

\title{
The Economic Order Quantity Repair and Waste Disposal Model: Solution Approaches
}

\author{
Misha Billah *a ${ }^{*}$ M. M. Rizvi ${ }^{\mathrm{a}, \mathrm{b}}$, and Lipon Chandra Das ${ }^{\mathrm{a}}$ \\ ${ }^{\mathrm{a}}$ Department of Mathematics, University of Chittagong, Chittagong-4331, Bangladesh. \\ ${ }^{\mathrm{b}}$ STEM-University of South Australia, Adelaide.
}

\begin{abstract}
As an inventory management instrument, Economic Order Quantity (EOQ) is gaining increased attention. EOQ has become the focus point of everyone's interest, especially scientists. There are inventory problems in the production and repair system where the used products are collected to repair, and after repairing, it is considered as a new product. A two-stage EOQ model for manufacturing, repairing and disposing of products is discussed in our analysis. The present model follows a former model introduced by Nahimas and Rivera, where the repair system is finite. We propose a new mathematical model in reverse logistics system where demand in production and repair items follows an exponential rate. Mathematical expressions for determining the production and repair quantities are also initiated here. In this model, it is assumed that the available space for supply and repair depots is limited, and we impose two constraints that turn the problem into a constrained optimization model. We also conduct extensive numerical experiments, and advantages are addressed.
\end{abstract}

(C) 2020 Published by Bangladesh Mathematical Society

Received: October 02, 2020 Accepted: December 29, 2020 Published Online: January 15, 2021

Keywords: EOQ model; Reverse logistics; Procurement; Repair; Exponential demands

AMS Subject Classifications 2020: 90B05; 90C30; 90B06

\section{Introduction}

Economic order quantity (EOQ) is the standard number of units that a company should purchase or produce to increase its inventory. EOQ is applied to minimize inventory holding costs and other related costs. Besides, reverse logistics refers to a process which relates to collecting and remanufacturing used products to extend their useable lives. Thus, reverse logistics helps us to reduce waste and conserve natural resources with remanufacturing being a collective noun for a process such as receiving, repairing and recycling products [1]. These inventories exist primarily to make goods available to customers or producers without delay and to increase sales and profits. It is natural to classify the type of items carried by an inventory process to two broad classifications, namely consumable and repairable items. For example, papers, pencils, fuels, food, etc. are considered consumable items that cannot be recycled or remanufactured. On the other hand, a repairable item

\footnotetext{
*Corresponding author. E-mail address: mishabillah123@gmail.com
} 
is one that can be repaired after a failure or wear-out and subsequently will provide some flow of services to the user. Automobiles, containers, engines, computers are all examples of repairable items [2].

After 1960, the repairable inventory was given importance for the first time. At that time, many models were published on economic order quantity. For determining optimal production and repair quantities, Schardy [2] first developed an inventory model. He proved that renovated products considered for moreover $50 \%$ of the currency staked in inventory. Schardy improved an economic order quantity model for fixable things. He assumed that the production and repair rates are abrupt without dumping costs. Schardy's model was extended by Nahimas and Rivera [3]. They considered a finite repair rate with the supposition of finite storage in new procurement and fix-it shops.

Reverse logistics came to be a business term in the $1990 \mathrm{~s}$ and the research on the inventory and manufacturing problem took a new trend. Richter [4]-[8] put a lot of effort to develop the EOQ model of the reverse logistics system and its properties. He investigated the cost analysis of his earlier works to show that a pure (bangbang) policy of either no waste disposal (total repair) or no repair (total waste disposal) dominates a mixed strategy of waste disposal and repair. He developed an EOQ model for stationary demand that is satisfied with production items of a certain product using new materials and components and from repairing used items that are collected from the market at the same rate. The EOQ repair and waste disposal problem was studied in [4-8] and was extended by Saadney and Jaber [9]-[13] to the problem of minimizing the total cost of production, remanufacturing and inventory. In the paper of Saadney and Jaber, the extended EOQ production; repair and waste disposal model of Richter was modified to show that ignoring the first time interval results in an unnecessary inventory and, consequently is an overestimation of the holding costs [4].

In our practical life, the demand for all daily necessities does not always stay constant. There are items the demand for which changes over time $t$. Such a situation has not much been discussed in the literature. In this paper, we try to establish a novel model that can describe a situation where demand change from time to time. In our analysis, considering that products or items decrease at an exponential rate with time $t$. We can utilize this concept in real-life situations to calculate optimum production or purchase in inventory management. More research can be seen in [14]-[19].

A two-stage EOQ model for a single product is considered in this project. Here we use two types of items; newly produced and repaired items to satisfy customer's demand. But after repairing, repaired items are also considered as good as new. At the first stage, we assume that $r$ is a recovery rate as a percentage of the demand $d=e^{-a t}$. We further assume that the repairable items are repaired at a constant rate of $\lambda$, and products are delivered from the repair depot to the supply depot with a constant rate of $\lambda-r e^{-a t}$. In the second stage, the inventory always decreases at a rate of $e^{-a t}$ to satisfy customer's demand.

The purpose of this paper is to investigate the repairable and disposal item inventory problem and to develop decision rules for a repairable inventory model giving due consideration to the costs associated with a repairable system and a waste disposal system.

The layout of the paper is as follows. The intended research has two stages, and these are the analytical part as well as the numerical experiments. In Section 2, in the mathematical part, we first provide a geometric model of the system. Then we develop the mathematical expressions for each movement of the item. In this section, we formulate a new mathematical model of the inventory problem. We establish the theoretical properties of the proposed model. In Section 3, we extend the proposed new model to the case where inventory cost is minimized by considering some constraints. In Section 4, we conduct computational experiments based on the theoretical results obtained in Sections 2 and 3.

\subsection{Assumptions}

In our analysis, we assume that a single product is procured and repaired items are considered as good as new. We further assume that lead time is zero, and no shortage is allowed, and we also consider that demands are known, constant and independent. Throughout the paper, we use the following notations.

$$
\begin{aligned}
& Q_{p} \rightarrow \text { quantity of new product in a single batch. } \\
& Q_{R} \rightarrow \text { quantity of repaired item in a single batch. } \\
& \tau_{p} \rightarrow \text { lead time of new product. } \\
& \tau_{R} \rightarrow \text { lead time of repaired item. } \\
& A_{p} \rightarrow \text { fixed cost of new product. } \\
& A_{R} \rightarrow \text { fixed cost of repaired item. }
\end{aligned}
$$




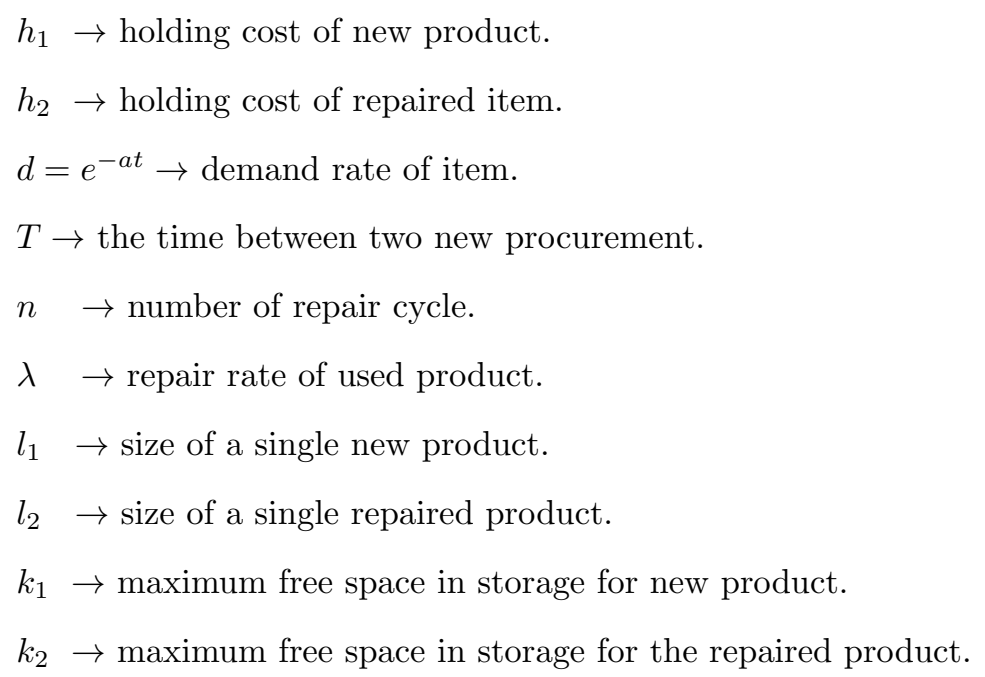

\section{$1.2 \quad$ Repairable Model}

In this model, we use two types of items. One of them is a new product and the other is a used-product. Used items are required to repair to meet customers' demand. In our study, we consider that after repairing, repaired products are sold as new products. The behavior of inventory for produced, collected used and repaired items over the total time interval $T$ is illustrated in Figure 1.1.

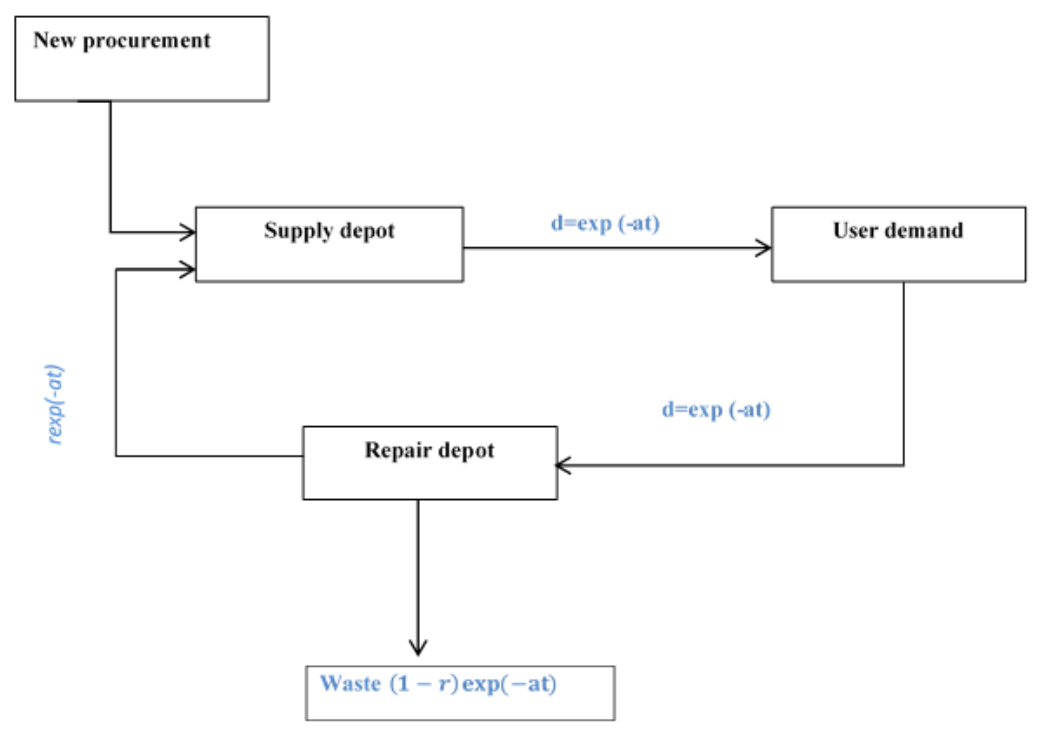

Figure 1.1: Material flow for manufacture and repair system.

\section{Mathematical Model}

Now we consider the case where the repair rate is finite. We also assume, when used products are recycled, then they are collected at the recycle store and gradually delivered in a fixed quantity to the supply store. Let the used items be repaired at finite rate, $\lambda>0$, always decrease at a rate of $e^{-a t}$. Similarly, Type- 2 inventory will always increase at a rate of $r e^{-a t}$. Since some used items are beyond repair, $(1-r) e^{-a t}$ items are condemned. 

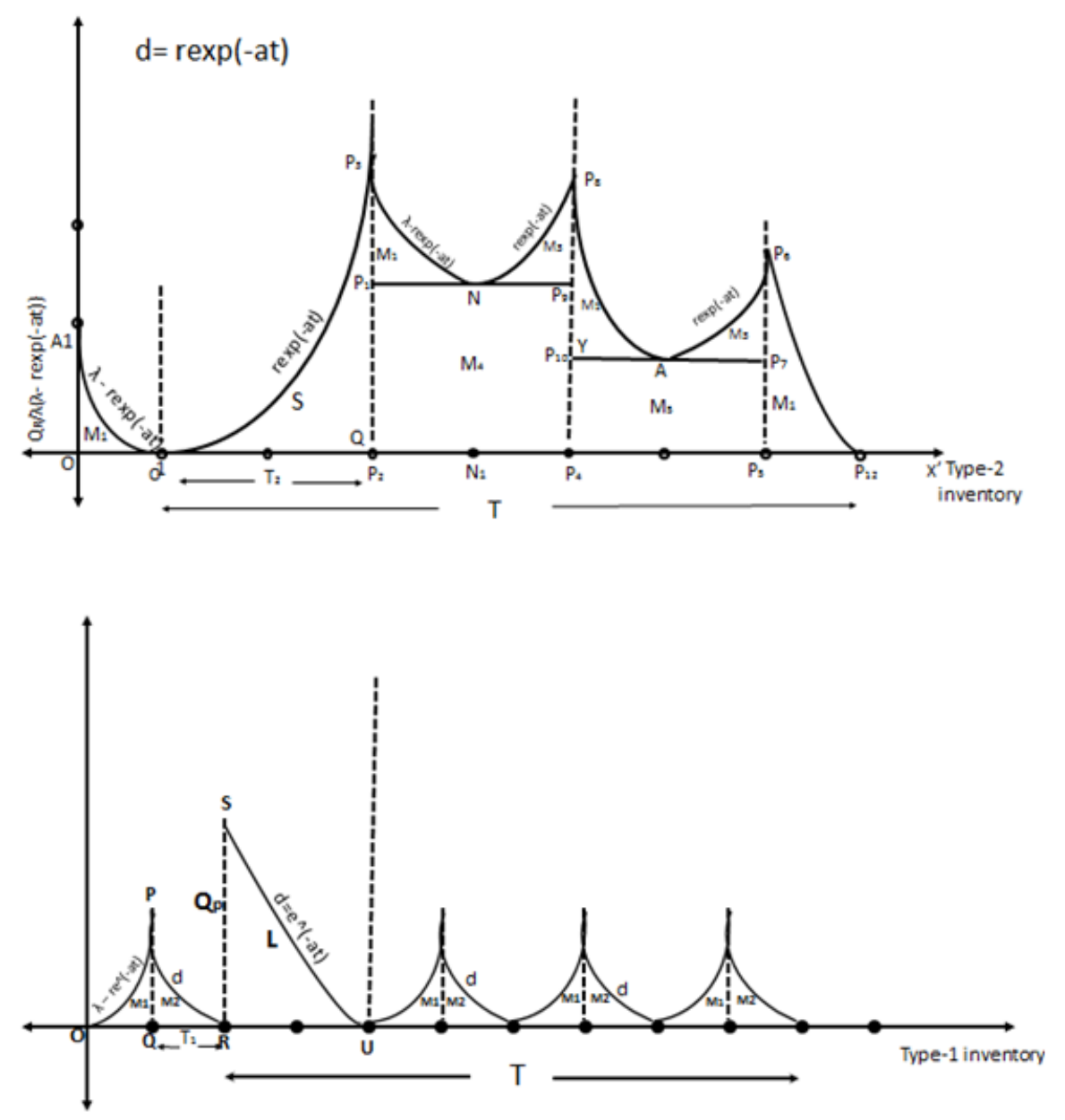

Figure 2.1: Repairable model with the exponential repair rate.

Again, we consider the repair batch is $Q_{R}$ that means at a time $Q_{R}$ items can be repaired in a batch. So during repair, the decrease in Type-2 inventory is $\frac{Q_{R}}{\lambda}\left(\lambda-r e^{-a t}\right)$.

The system is shown in Figure-2.1. From the figure, we can say the procurement and repair lead times $\tau_{p} \& \tau_{R}$ are zero for clarity of presentation.

At the same time, Type- 1 inventory gradually increased at a rate of $\left(\lambda-r e^{-a t}\right)$. So from Type-2 inventory $\frac{Q_{R}}{\lambda}\left(\lambda-r e^{-a t}\right)$ items are delivered to Type-1 in a single batch. So we can write $\left(\lambda-r e^{-a t}\right)=\frac{Q_{R}}{\lambda}\left(\lambda-r e^{-a t}\right)+$ $c_{1}$, this yields, $t=\frac{1}{a} \log \frac{r\left(\lambda-Q_{R}\right)}{\lambda\left(\lambda-Q_{R}-c_{1}\right)}$.

Thus, the area of OPQ is $\int_{0}^{\frac{1}{a} \log \frac{r\left(\lambda-Q_{R}\right)}{\lambda\left(\lambda-Q_{R}-c_{1}\right)}}\left(\lambda-r e^{-a t}\right) d t$, and thus

$$
[\lambda t]_{0}^{\frac{1}{a} \log \frac{r\left(\lambda-Q_{R}\right)}{\lambda\left(\lambda-Q_{R}-c_{1}\right)}}+\frac{r}{a}\left[e^{-a t}\right]_{0}^{\frac{1}{a} \log \frac{r\left(\lambda-Q_{R}\right)}{\lambda\left(\lambda-Q_{R}-c_{1}\right)}},
$$

therefore, we have

$$
M_{1}=\frac{\lambda}{a} \log \frac{r\left(\lambda-Q_{R}\right)}{\lambda\left(\lambda-Q_{R}-c_{1}\right)}+\frac{r}{a}\left(\frac{\lambda\left(\lambda-Q_{R}-c_{1}\right)}{r\left(\lambda-Q_{R}\right)}-1\right) .
$$

In Type-1 inventory, products are delivered to the customers at a rate of $e^{-a t}$. At the point, when the repaired 
products are exhausted, we bring a new procurement batch $Q_{p}$ to satisfy customers' demand. While the repair process continues in repair depot system, from Figure 2.1, we get $e^{-a t}=Q_{p}+c_{1}$, thus, $t=-\frac{1}{a} \log \left(Q_{p}+c_{1}\right)$.

The area of PQR is

$$
M_{2}=\int_{\frac{1}{a}}^{-\frac{1}{a} \log \left(Q_{p}+c_{1}\right)} \frac{r\left(\lambda-Q_{R}\right)}{\lambda\left(\lambda-Q_{R}-c_{1}\right)} e^{-a t} d t
$$

this implies,

$$
M_{2}=\frac{1}{a}\left\{\frac{\lambda\left(\lambda-Q_{R}-c_{1}\right)}{r\left(\lambda-Q_{R}\right)}-\left(Q_{p}+c_{1}\right)\right\} .
$$

When the new procurement item in Type-2 inventory reaches tends to zero, again we bring repaired items from Type-1 inventory. Repaired items again come to the supply depot system at a rate of $\left(\lambda-r e^{-a t}\right)$. It follows that, $e^{-a t}=c_{1}$, it yields, $t=-\frac{1}{a} \log c_{1}$.

So, the area of RSU is

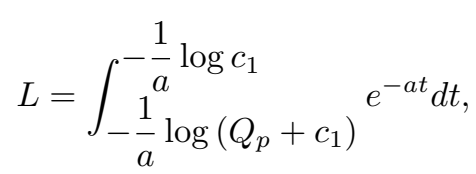

and therefore, $L=\frac{1}{a} Q_{p}$. Now, the stated process will continue to repeat until the new procurement (which is also equal to the time between successive suspensions of repair) arrives. The time of cycle length $T$ is equal to two new procurements. Thus, we get the value of $T$ from Figure-2.1. Here,

$$
T=-\frac{1}{a} \log c_{1}+(n-1)\left(-\frac{1}{a} \log \left(Q_{p}+c_{1}\right)\right),
$$

where $n$ is the value of total repair inductions in a cycle.

In order to find the total supply depot area, it is necessary to find the value of $n$. From Type- 2 inventory, the value of $n$ can be determined by successive inductions due to condemnations. So the successive repair induction is $p_{1} p_{3}-p_{8} p_{9}$ or $\left\{r c_{1}\left(1-Q_{p}-c_{1}\right)\right\}$.

Thus, the total loss of stock is $(n-1) r c_{1}\left(1-Q_{p}-c_{1}\right)$, which must be equal to the value of $\left(p_{2} p_{3}-p_{6} p_{5}\right)$ or $\left(r c_{1}-Q_{R}\right)$. Therefore, we can write, $(n-1) r c_{1}\left(1-Q_{p}-c_{1}\right)=\left(r c_{1}-Q_{R}\right)$, this implies,

$$
n=\frac{\left(r c_{1}-Q_{R}\right)}{r c_{1}\left(1-Q_{p}-c_{1}\right)}+1 .
$$

Thus, we get the actual value of $T$, and we illustrate this as

$$
T=-\frac{1}{a} \log c_{1}-\frac{1}{a} \frac{\left(r c_{1}-Q_{R}\right)}{r c_{1}\left(1-Q_{p}-c_{1}\right)} \log \left(Q_{p}+c_{1}\right) .
$$

Let $A_{1}$ be the area of the Type-1 inventory curve during a cycle $T$. So from the Figure-2.1, we can write, $A_{1}=L+n\left(M_{1}+M_{2}\right)$, thus we have

$$
A_{1}=\frac{Q_{p}}{a}+\left(\frac{\left(r c_{1}-Q_{R}\right)}{r c_{1}\left(1-Q_{p}-c_{1}\right)}+1\right)\left[\frac{\lambda}{a}\left\{\log \frac{r\left(\lambda-Q_{R}\right)}{\lambda\left(\lambda-Q_{R}-c_{1}\right)}+\frac{(r+1)\left(\lambda-Q_{R}-c_{1}\right)}{r\left(\lambda-Q_{R}\right)}\right\} \frac{1}{a}\left(r+Q_{p}+c_{1}\right)\right] .
$$

Let $A_{2}$ be the area under Type-2 inventory curve during one cycle. Now we can divide the area $A_{2}$ into some small areas and rectangles, that help us to calculate the total area. By comparing with Type- 2 inventory, we see the limit of area $O^{\prime} p_{2} p_{3}$ from $t=\frac{1}{a} \log \frac{r\left(\lambda-Q_{R}\right)}{\lambda\left(\lambda-Q_{R}-c_{1}\right)}$ to $t=-\frac{1}{a} \log c_{1}$.

So, the area of $O^{\prime} p_{2} p_{3}$ is

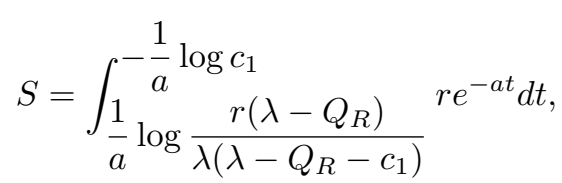


this yields,

$$
S=\frac{r}{a}\left(\frac{\lambda\left(\lambda-Q_{R}-c_{1}\right)}{r\left(\lambda-Q_{R}\right)}-c_{1}\right)
$$

The area of $p_{1} p_{3} N, p_{8} p_{10} A, p_{5} p_{6} p_{12}$ are equal to the area of $O P Q$ in Figure-2.1. From Figure-2.1, we find the limit of area $p_{8} p_{9} N$ is $\left(-\frac{1}{a} \log c_{1}+\frac{1}{a} \log \frac{r\left(\lambda-Q_{R}\right)}{\lambda\left(\lambda-Q_{R}-c_{1}\right)}\right)$ to $\left(-\frac{1}{a} \log c_{1}-\frac{1}{a} \log \left(Q_{p}+c_{1}\right)\right)$. So the area of $p_{8} p_{9} N$ is

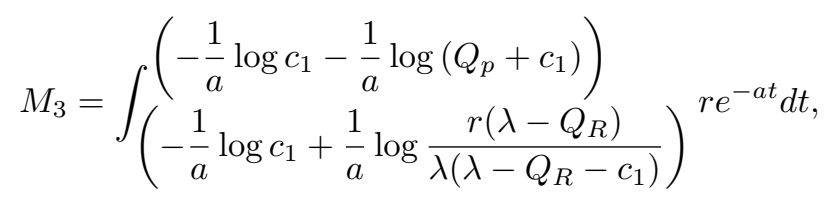

and thus,

$$
M_{3}=\frac{r}{a}\left\{\frac{c_{1} \lambda\left(\lambda-Q_{R}-c_{1}\right)}{r\left(\lambda-Q_{R}\right)}-c_{1}\left(Q_{p}+c_{1}\right)\right\} .
$$

Now, we have to find the area of rectangles $p_{1} p_{2} p_{4} p_{9} \& p_{4} p_{5} p_{7} p_{10}$. Therefore, rectangle $p_{2} p_{4} p_{9} p_{1}$ has a base, $p_{2} p_{4}=-\frac{1}{a} \log \left(Q_{p}+c_{1}\right)$, and height, $p_{2} p_{1}=p_{2} p_{3}-p_{1} p_{3}$, where, $p_{2} p_{3}+c_{2}=r e^{\left(-a\left(-\frac{1}{a} \log c_{1}\right)\right)}$. Thus, $p_{2} p_{3}=$ $r c_{1}-c_{2}$, and $p_{2} p_{1}=\left\{\left(r c_{1}-c_{2}\right)-\frac{Q_{R}}{\lambda}\left(\lambda-r e^{-a t}\right)\right\}$. Therefore, the area of $p_{2} p_{4} p_{9} p_{1}$ is $M_{4}=p_{2} p_{1} \times p_{2} p_{4}$, so,

$$
M_{4}=\frac{1}{a} \log \left(Q_{p}+c_{1}\right)\left\{\frac{Q_{R}}{\lambda}\left(\lambda-r e^{-a t}\right)-\left(r c_{1}-c_{2}\right)\right\} .
$$

Rectangle $p_{4} p_{5} p_{7} p_{10}$ has the same base of the rectangle $p_{1} p_{2} p_{4} p_{9}$. But height is $p_{4} p_{10}$ which is equal to $\left(p_{4} p_{8}-p_{8} p_{10}\right)$.

Now, $p_{8} p_{10}=p_{1} p_{3}=\frac{Q_{R}}{\lambda}\left(\lambda-r e^{-a t}\right)$, where, $p_{4} p_{8}+c_{2}=r c_{1}\left(Q_{p}+c_{1}\right)$. So, $p_{4} p_{10}=r c_{1}\left(Q_{p}+c_{1}\right)-$ $\frac{Q_{R}}{\lambda}\left(\lambda-r e^{-a t}\right)-c_{2}$. Thus, the area of $p_{4} p_{5} p_{7} p_{10}$ is $M_{5}=p_{4} p_{5} \times p_{4} p_{10}$, this implies,

$$
M_{5}=\frac{1}{a} \log \left(Q_{p}+c_{1}\right)\left\{r c_{1}\left(Q_{p}+c_{1}\right)-c_{2}-\frac{Q_{R}}{\lambda}\left(\lambda-r e^{-a t}\right)\right\} .
$$

So, the total repair depot area is,

$$
\begin{aligned}
A_{2}= & S+M_{4}+M_{5}+n M_{1}+(n-1) M_{3} \\
= & \frac{r}{a}\left(\frac{\lambda\left(\lambda-Q_{R}-C_{1}\right)}{r\left(\lambda-Q_{R}\right)}-c_{1}\right)+\frac{1}{a} \log \left(Q_{p}+c_{1}\right)\left\{\frac{Q_{R}}{\lambda}\left(\lambda-r e^{-a t}\right)-\left(r c_{1}-c_{2}\right)\right\} \\
& +\frac{1}{a} \log \left(Q_{p}+c_{1}\right)\left\{r c_{1}\left(Q_{p}+c_{1}\right)-c_{2}-\frac{Q_{R}}{\lambda}\left(\lambda-r e^{-a t}\right)\right\} \\
& +\left(\frac{\left(r c_{1}-Q_{R}\right)}{r c_{1}\left(1-Q_{p}-c_{1}\right)}+1\right)\left\{\frac{\lambda}{a} \log \frac{r\left(\lambda-Q_{R}\right)}{\lambda\left(\lambda-Q_{R}-c_{1}\right)}+\frac{r}{a}\left(\frac{\lambda\left(\lambda-Q_{R}-c_{1}\right)}{r\left(\lambda-Q_{R}\right)}-1\right)\right\} \\
& +\frac{\left(r c_{1}-Q_{R}\right)}{r c_{1}\left(1-Q_{p}-c_{1}\right)} \frac{r}{a}\left\{\frac{c_{1}\left(\lambda-Q_{R}-c_{1}\right)}{r\left(\lambda-Q_{R}\right)}-c_{1}\left(Q_{p}+c_{1}\right)\right\},
\end{aligned}
$$

After simplifications, we get

$$
\begin{aligned}
A_{2}= & \frac{2 \lambda}{a} \frac{\left(\lambda-Q_{R}-c_{1}\right)}{\left(\lambda-Q_{R}\right)}-\frac{r}{a}\left(1+c_{1}\right)+\frac{1}{a} \log \left(Q_{p}+c_{1}\right)\left\{r c_{1}\left(Q_{p}+c_{1}-1\right)\right\} \\
& +\frac{r c_{1}-Q_{R}}{r c_{1}\left(1-Q_{p}-c_{1}\right)}\left[\frac{\lambda}{a} \log \frac{r\left(\lambda-Q_{R}\right)}{\left(\lambda-Q_{R}-c_{1}\right)}+\frac{\lambda}{a} \frac{\left(\lambda-Q_{R}-c_{1}\right)}{\left(\lambda-Q_{R}\right)}\left(1+c_{1}\right)-\frac{r}{a}\left\{1+c_{1}\left(Q_{p}+c_{1}\right)\right\}\right] \\
& +\frac{\lambda}{a} \log \frac{r\left(\lambda-Q_{R}\right)}{\left(\lambda-Q_{R}-c_{1}\right)},
\end{aligned}
$$

It now follows that the total cost $c\left(Q_{R}, Q_{p}\right)$ is given by 


$$
\begin{aligned}
& c\left(Q_{R}, Q_{p}\right)=A_{p}+n A_{R}+h_{1} A_{1}+h_{2} A_{2} \\
&=A_{p}+\left(\frac{\left(r c_{1}-Q_{R}\right)}{r c_{1}\left(1-Q_{p}-c_{1}\right)}+1\right) A_{R} \\
& \quad+h_{1}\left[\frac{Q_{p}}{a}+\left(\frac{\left(r c_{1}-Q_{R}\right)}{r c_{1}\left(1-Q_{p}-c_{1}\right)}+1\right) \frac{\lambda}{a}\left(\log \frac{r\left(\lambda-Q_{R}\right)}{\lambda\left(\lambda-Q_{R}-c_{1}\right)}+\frac{(r+1)\left(\lambda-Q_{R}-c_{1}\right)}{r\left(\lambda-Q_{R}\right)}\right)-\frac{1}{a}\left(r+Q_{p}+c_{1}\right)\right] \\
&+h_{2}\left[\frac{2 \lambda}{a} \frac{\left(\lambda-Q_{R}-c_{1}\right)}{\left(\lambda-Q_{R}\right)}-\frac{r}{a}\left(1+c_{1}\right)+\frac{1}{a} \log \left(Q_{p}+c_{1}\right)\left\{r c_{1}\left(Q_{p}+c_{1}-1\right)\right\}\right. \\
&+\frac{r c_{1}-Q_{R}}{r c_{1}\left(1-Q_{p}-c_{1}\right)}\left[\frac{\lambda}{a} \log \frac{r\left(\lambda-Q_{R}\right)}{\left(\lambda-Q_{R}-c_{1}\right)}+\frac{\lambda}{a} \frac{\left(\lambda-Q_{R}-c_{1}\right)}{\left(\lambda-Q_{R}\right)}\left(1+c_{1}\right)-\frac{r}{a}\left\{1+c_{1}\left(Q_{p}+c_{1}\right)\right\}\right] \\
&\left.+\frac{\lambda}{a} \log \frac{r\left(\lambda-Q_{R}\right)}{\left(\lambda-Q_{R}-c_{1}\right)}\right] .
\end{aligned}
$$

And the average cost is given by

$$
K\left(Q_{R}, Q_{p}\right)=\frac{c\left(Q_{R}, Q_{p}\right)}{T}=c\left(Q_{R}, Q_{p}\right)\left[-\frac{1}{a} \log c_{1}-\frac{1}{a} \frac{\left(r c_{1}-Q_{R}\right)}{r c_{1}\left(1-Q_{p}-c_{1}\right)} \log \left(Q_{p}+c_{1}\right)\right]^{-1} .
$$

\section{Extension}

We can extend the above model by imposing constraints on available space in the supply and repair depots area. Let $k_{1}$ and $k_{2}$ are the maximum free space in storage for new and repaired products, respectively. We also know that $Q_{p}$ is the size of new procurement at unit time in Type-1 inventory. From Type-2 inventory, we get the value of $p_{2} p_{3}$ is $\left(r c_{1}-c_{2}\right)$ or $r c_{1}\left(1-Q_{R} / \lambda\right)$, where $Q_{R}$ is the size of the repaired procurement at unit time. So, we can express the model as follows:

minimize $f(x)=K\left(Q_{R}, Q_{p}\right)$. subject to the conditions,

$$
\left\{\begin{array}{l}
l_{1} Q_{p} \leq k_{1} \\
l_{2} r c_{1}\left(1-Q_{R} / \lambda\right) \leq k_{2},
\end{array}\right.
$$

where $l_{1}$ and $l_{2}$ are the size of a single new and repaired product, respectively.

\section{Numerical Illustration}

In this section, we first test the proposed cost function (2.1) for fixed input parameter values.

\subsection{Test Problem: 1}

Let us set the input parameters as $a=.5 ; c_{1}=.3 ; \lambda=20 ; r=.5 ; A_{p}=1 ; A_{r}=1 ; h_{1}=.6 ; h_{2}=.2$; We aim to minimize function $K\left(Q_{R}, Q_{p}\right)$, under the lower bound $Q_{p} \geq 5$ and $Q_{R} \geq 22$. In these setting, we minimize the function (2.1) and obtain the procurement and repair batches are $Q_{p}=10.7040$ and $Q_{R}=22$ with the optimal cost 9.5382. The solution and function surface of (2.1) are depicted in Figure 4.1. We solved Test Problem 1 writing code in MATLAB. We utilize a range of nonlinear solvers such as fmincon with sequential quadratic programming, Ipopt [20], and SCIP [21]. We have got an approximation of the solution set that is demonstrated in Figure 4.1 (red circles). 


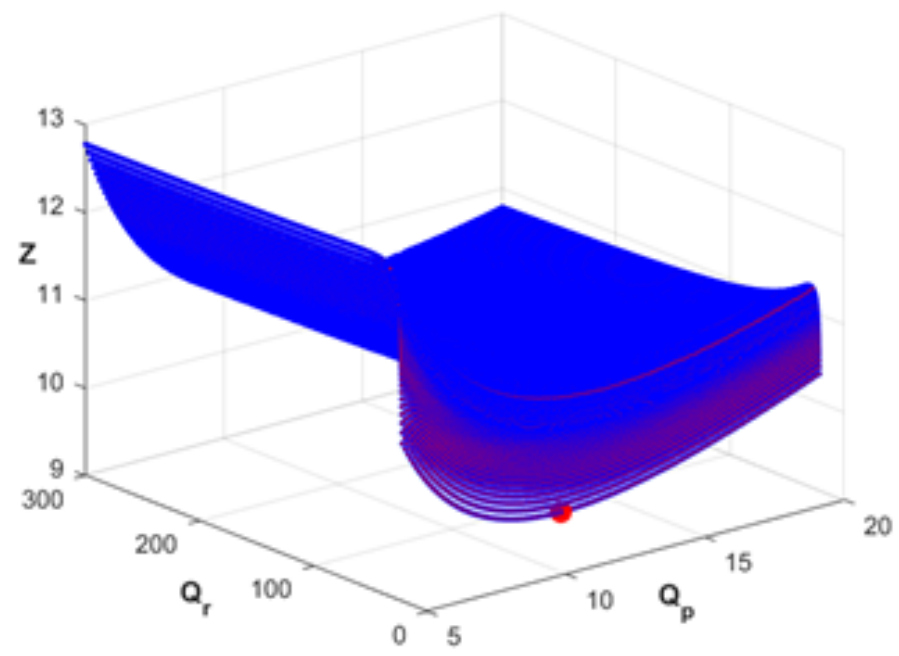

Figure 4.1: The circle (red) depicts the solution of Test problems $1 \& 2$, and blue surface demonstrates the geometric surface of (2.1) and (3.1).

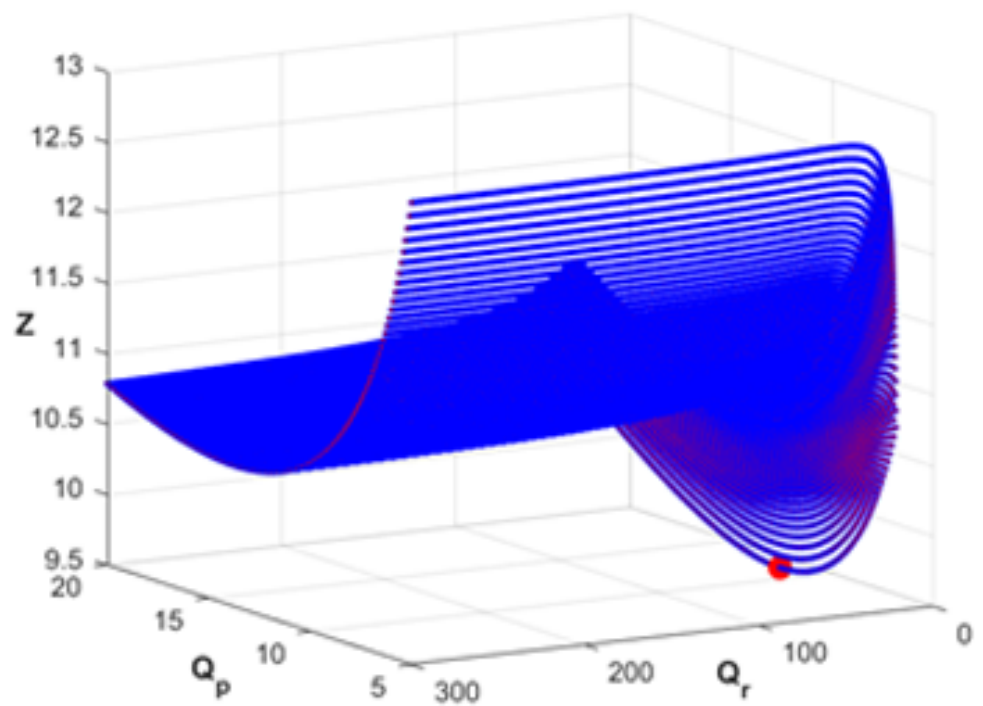

Figure 4.2: Rotated view of Figure 4.1

\subsection{Test Problem: 2}

Let us set the parameters as $a=.5 ; c_{1}=.3 ; \lambda=20 ; r=.5 ; A_{p}=1 ; A_{r}=1 ; h_{1}=.6 ; h_{2}=.2 ; l_{1}=.3$; $l_{2}=.5 ; k_{1}=160 ; k_{2}=120 ;$ We aim to minimize function $K\left(Q_{R}, Q_{p}\right)$, subject to constraints (3.1) under the lower bound $Q_{p} \geq 5$ and $Q_{R} \geq 22$. We utilize the same algorithm that we were considered to solve Test Problem 1. We observe that we obtain the same result, as demonstrated in Figure 4.1.

Open Question: The above model can be extended to the problem where demand is stochastic, implies that, demand is not known in advance. In this case, demand always cannot be fulfilled by the supply system where the shortage is allowed. The model can also be extended to the hybrid problem, which is a mixture of both deterministic and stochastic. We leave this research as our future project. 


\section{Conclusions}

We proposed a noble mathematical model of the reverse logistics system. The model extended Nahimas approach where we considered that the demands for procured and repaired items are exponential with time. We developed two-phase mathematical models to minimize inventory costs. The proposed first model optimizes the cost that is not restricted by any conditions. The second model includes constraints on floor space. This turns the problem into a constrained optimization problem. Both models find the optimum procurement and repair batches such that the total cost would be minimal. We expect our established mathematical model in reverse logistics can solve many real-life complex problems. Numerical experiments were also conducted to test the models. Moreover, we provided two examples with the same input parameters and solved the problem using a range of nonlinearly constrained optimization solvers.

\section{References}

[1] M. A. Ahmed and El Saadney, M. Y. Jaber, A Production/Remanufacturing inventory model with price and quality dependent return rate, Computer \& Industrial Engineering, 58: 352-362 (2010).

[2] D. A. Schardy, A deterministic inventory model for repairable items, Naval Research Logistics, 14(2): 391-398 (1967).

[3] S. Nahimas and H. Rivera, A deterministic model for a repairable item inventory system with a finite repair rate, International Journal of Production Research, 17(3): 215-221 (1979).

[4] K. Richter, The EOQ repair and waste disposal model with variable setup numbers, European Journal of Operational Research, 95(2): 313-324 (1996a).

[5] K. Richter, The extended EOQ repair and waste disposal model, International Journal of production of Economics, 45(1-3): 443-447 (1996b).

[6] K. Richter, An EOQ repair and waste disposal model, Preprints of the 8th International Working Seminar on Production Economics, Innsbruck, 3: 83-91 (1994).

[7] K. Richter, Pure and mixed strategies for the EOQ repair and waste disposal problem, OR Spektrum, 19(2): 123-129, (1999).

[8] K. Richter and I. Dobos, Analysis of the EOQ repair and waste disposal problem with integer setup numbers, International Journal of Production Economics, 59(1-3): 463-467 (1999).

[9] M. Y. Jaber and M. A. Rosen, The economic order quantity repair and waste disposal model with entropy cost, European Journal of Operational Research, 188(1): 109-120 (2008).

[10] M. Y. Jaber and A. M. A. EI Saadney, The production, remanufacture and waste disposal model with lost sales, Int. J. Production Economics, 120: 115-124 (2009).

[11] A. M. A. EI Saadney and M. Y. Jaber, A production/remanufacturing inventory model with price and quality dependent return rate, Computers \& Industrial Engineering, 58: 352-362 (2010).

[12] A. M. A. EI Saadney and M. Y. Jaber, How many times to remanufacture? International Journal of Production Economics, 143(2): 598-604 (2013).

[13] M. Y. Jaber, C. H. Glock and A. M. A. EI Saadney, Supply chain coordination with emissions reduction incentives, International Journal of Production Research, 51(1): 69-82 (2013a).

[14] I. Dobos and K. Richter, The integer EOQ repair and waste disposal model - Further analysis, Central European Journal of Operations Research, 8(2): 173-194 (2000).

[15] I. Dobos and K. Richter, A production/recycling model with stationary demand and return rates, Central European Journal of Operations Research, 11(1): 35-46 (2003).

[16] I. Dobos and K. Richter, K, An extended production/recycling model with stationary demand and return rates, International Journal of Production Economics, 90(3): 311-323 (2004). 
[17] I. Dobos and K. Richter, A production/recycling model with quality consideration, International Journal of Production Economics, 104(2): 571-579 (2006).

[18] N. Kozlovskaya, K. Pakhomova, and K. Richter, A note on "The EOQ repair and waste disposal model with switching costs", Computers \& Industrial Engineering, 103. pp. 310-315, 2017.

[19] E. Bazan, M. Y. Jaber, and S. Zanoni, A review of mathematical inventory models for reverse logistics and the future of its modeling: An environmental perspective, Applied Mathematical Modelling, 40: 4151-4178 (2016).

[20] A. Wachter and L. T. Biegler, On the implementation of a primal-dual interior point filter line search algorithm for large-scale nonlinear programming, Mathematical Programming, 106(1): 25-57, (2006).

[21] T. Achterberg, SCIP: Solving constraint integer programs, Mathematical Programming Computation, 1(1): 1-41 (2009).

\section{APPENDIX A}

Code is written in MATLAB for Examples given in the text. We have included the code in the text if anyone is interested in running the algorithms to solve any test problems.

\section{Listings}

1 MATLAB function file for objective function . . . . . . . . . . . . . . . . . . . . . 143

2 MATLAB function file for constraints . . . . . . . . . . . . . . . . . . . . . . . 143

3 MATLAB directory file to solve the problem . . . . . . . . . . . . . . . . . . . . . 143

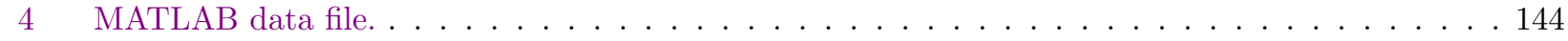

Listing 1: MATLAB function file for objective function

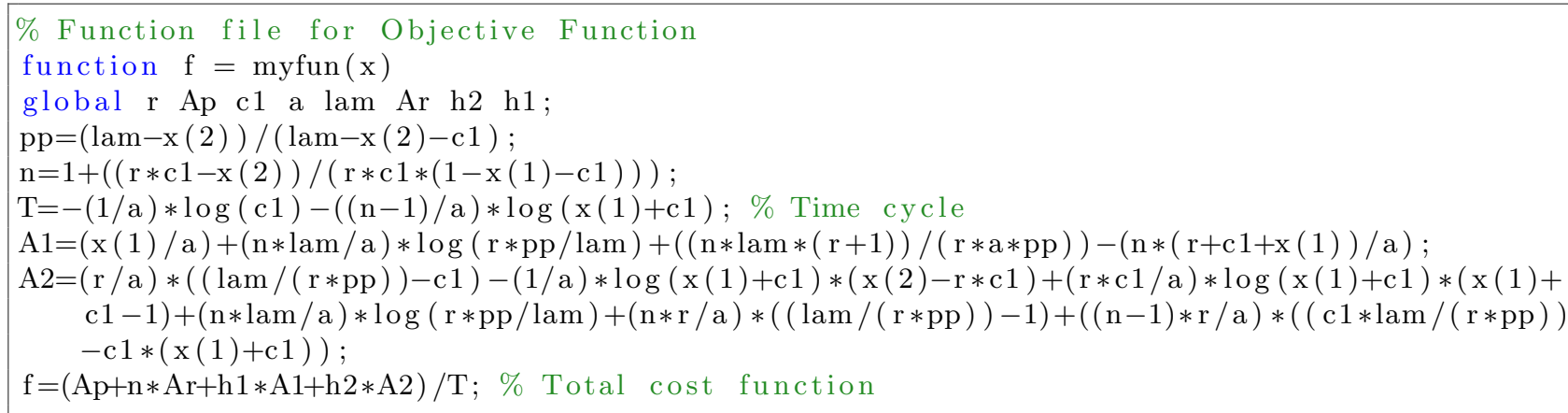

Listing 2: MATLAB function file for constraints

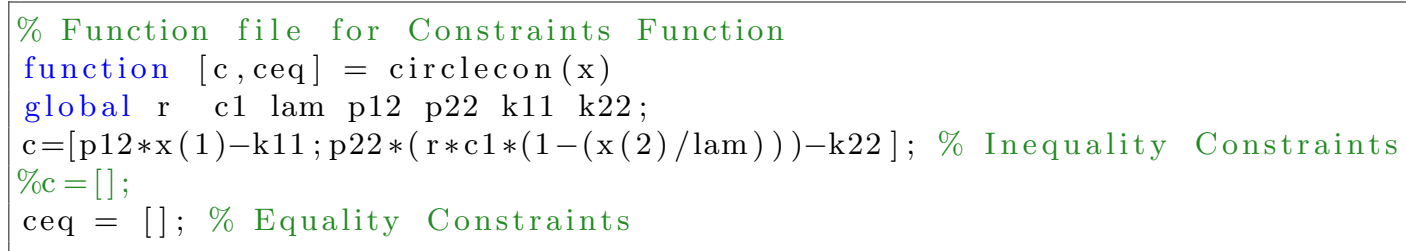

Listing 3: MATLAB directory file to solve the problem

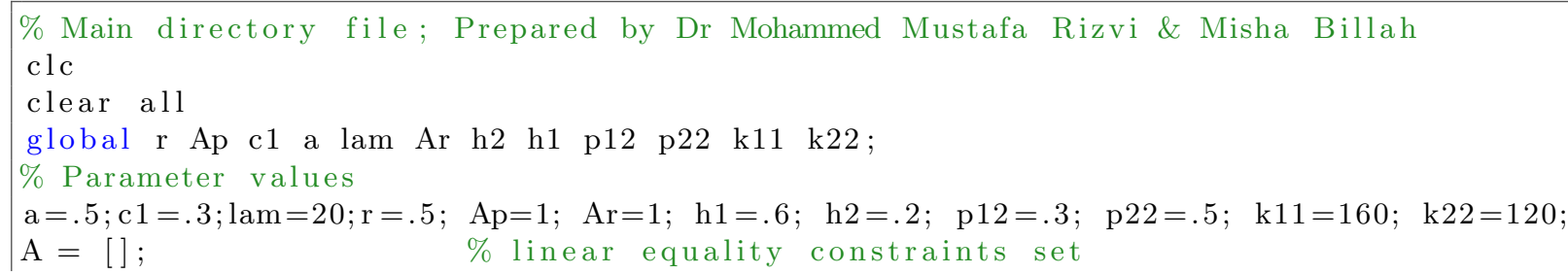




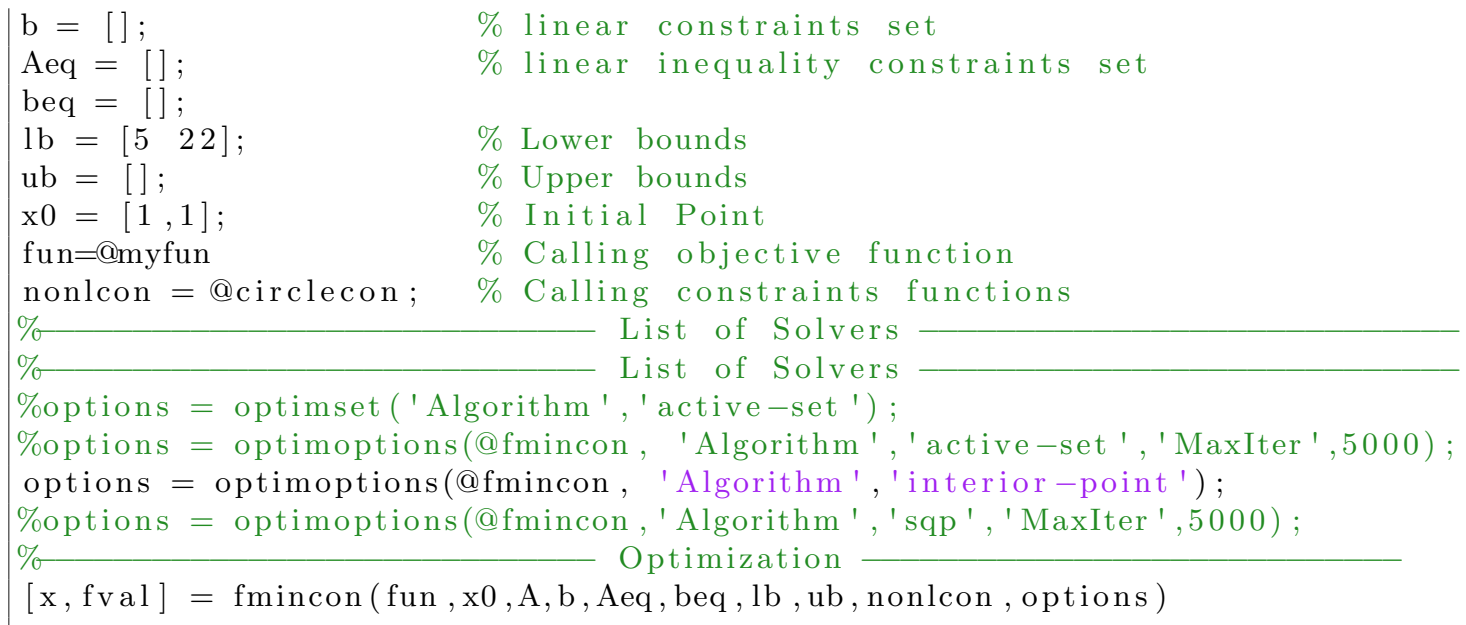

Listing 4: MATLAB data file.

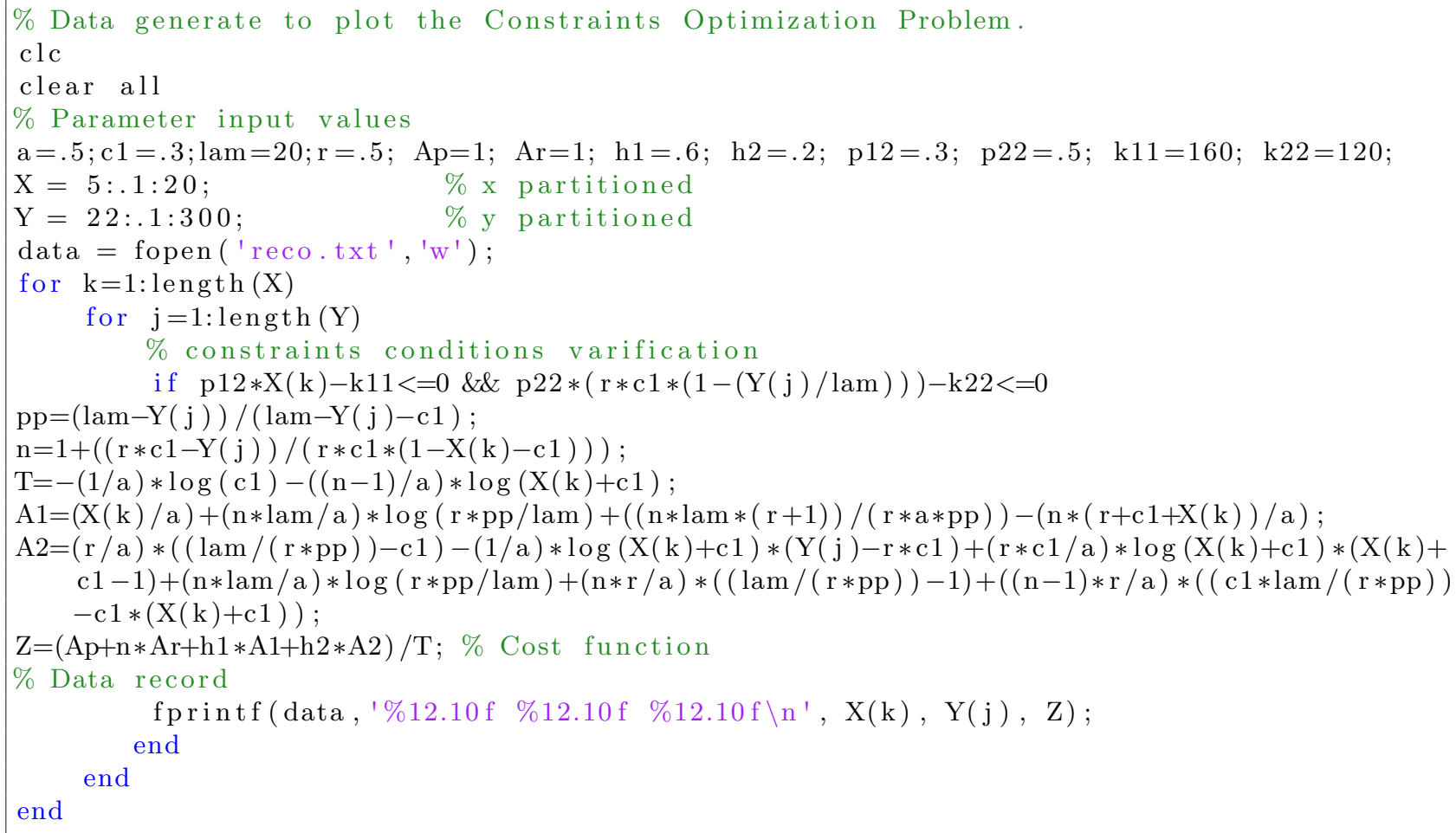

